 \\ TATRA \\ MOUNTaiNS \\ Mathematical Publications
}

\section{SET OF CONTINUITY POINTS OF FUNCTIONS WITH VALUES IN GENERALIZED METRIC SPACES}

\author{
Lubica Holá - Zbigniew Piotrowski
}

\begin{abstract}
We study continuity points of functions with values in generalized metric spaces. We define the generalized oscillation, which is a useful tool in our study. Let $X$ be a topological space and $Y$ be a weakly developable space. Let $f: X \rightarrow Y$ be a function. Then the set $C(f)$ of continuity points of $f$ is a $G_{\delta}$-set in $X$. Some results concerning continuity points of separately continuous functions as well as functions with closed graphs are also given.
\end{abstract}

\section{Introduction}

It is well-known that the set of points of discontinuity of functions with values in metrizable spaces belongs to the class $F_{\sigma}$ of countable unions of closed sets.

Of course, a natural question arises for which "larger" class of spaces the above assertion still holds. A quite natural candidate is the class of developable spaces as it was proved in GP1]. In our paper we prove that every function with values in a weakly developable space also has a $G_{\delta}$-set of continuity points.

Weakly developable spaces were introduced by J. Calbrix and B. Alle $\mathrm{ch}$ e in $\mathrm{CA}$. It was proved in $\mathrm{AAC}$ that a completely regular space is weakly developable if and only if it is a $p$-space with a $G_{\delta}$-diagonal.

Moreover, we prove that a topological space $Y$ having the property:

$(\triangle)$ given a topological space $X$ and $f: X \rightarrow Y$ the set $C(f)$ of the points of continuity of $f$ is a $G_{\delta}$-set in $X$,

has a $G_{\delta}$-diagonal.

Some results concerning continuity points of separately continuous functions and functions with closed graphs are also given.

2000 Mathematics Subject Classification: Primary 54C30; Secondary 54E18, 54 E50. Keywords: $p$-space, $G_{\delta}$-diagonal, developable space, weakly developable space, quasicontinuous function, generalized oscillation. 


\section{LUBICA HOLÁ - ZBIGNIEW PIOTROWSKI}

\section{Preliminaries}

All spaces are assumed to be Hausdorff. We quote $\mathrm{E}$ and $\mathrm{Gr}$ as basic references.

Let $Y$ be a topological space, $y \in Y$ and $\mathcal{G}$ be a collection of subsets of $Y$. Then

$$
s t(y, \mathcal{G})=\bigcup\{G \in \mathcal{G}: y \in G\} .
$$

Let $\left\{\mathcal{G}_{n}: n \in \omega\right\}$ be a sequence of open covers of $Y$.

(1) If for each $y \in Y$, the set $\left\{s t\left(y, \mathcal{G}_{n}\right): n \in \omega\right\}$ is a base at $y$, we say that $\left\{\mathcal{G}_{n}: n \in \omega\right\}$ is a development on $Y$ and that the space $Y$ is developable. A regular developable space is called a Moore space.

(2) If for every sequence $\left\{G_{n}: n \in \omega\right\}$ such that $G_{n} \in \mathcal{G}_{n}$ for every $n \in \omega$ and for every $y \in \cap G_{n}$, the sequence $\left\{\cap_{i \leq n} G_{i}: n \in \omega\right\}$ is a base at $y$, we say that $\left\{\mathcal{G}_{n}: n \in \omega\right\}$ is a weak development on $Y$ and that the space $Y$ is weakly developable.

The notion of a weak development was introduced by B. A lle ch e and J. C a lbrix in CA]. Notice that in the paper BLL of D. Burke, D. Lutzer and S. Levi, there is a notion (without a name) very close to the notion of a weak development; they consider a sequence of open covers $\left\{\mathcal{G}_{n}: n \in \omega\right\}$ on a completely regular space $Y$ such that for every $\left\{G_{n}: n \in \omega\right\}, G_{n} \in \mathcal{G}_{n}$ for every $n \in \omega$, every $y \in \cap G_{n}$, the sequence $\left\{\cap_{i \leq n} \overline{G_{i}}: n \in \omega\right\}$ is a base at $y$. It is easy to see that, in the class of regular spaces, the notion of [BLL] coincides with the notion of a weak development.

Of course, every developable space is weakly developable and every weakly developable space has a $G_{\delta^{-}}$diagonal (see [AAC]).

An example of a weakly developable space which is not developable is $\mathrm{G} \mathrm{r} \mathrm{u} \mathrm{-}$ e $n$ h a g e's space Gr] (see [AAC]).

By a result of AAC, a completely regular space is weakly developable if and only if it is a $p$-space with a $G_{\delta}$-diagonal.

A completely regular space $Y$ is a $p$-space if there exists a sequence

$$
\left\{\mathcal{G}_{n}: n \in \omega\right\}
$$

of families of open subsets of the Čech-Stone compactification $\beta Y$ such that:

(i) each $\mathcal{G}_{n}$ covers $Y$;

(ii) for each $y \in Y, \cap\left\{s t\left(y, \mathcal{G}_{n}\right): n \in \omega\right\} \subset Y$.

It is known (see $\mathrm{Gr}$ ) that Gruenhage's space is locally compact and submetrizable, i.e., it is weakly developable. It is not a Moore space since it contains a closed set which is not a $G_{\delta}$-set. 
The notion of so-called generalized oscillation is a useful tool in our investigation. Notice that the idea to define a generalized oscillation is not new in literature, see, for example, [P1], where the different approach from our is used.

Let $Y$ be a weakly developable space, $X$ be a topological space and $f: X \rightarrow Y$ be a function. Let $\left\{\mathcal{G}_{n}: n \in \omega\right\}$ be a weak development on $Y$. Of course, without loss of generality, we can suppose that the sequence $\left\{\mathcal{G}_{n}: n \in \omega\right\}$ is such that

$$
\mathcal{G}_{1}=\{Y\} \quad \text { and } \quad \mathcal{G}_{n+1} \prec \mathcal{G}_{n} \quad \text { for every } n \in \omega .
$$

(If $\mathcal{U}, \mathcal{V}$ are two collections of subsets of $Y$, by $\mathcal{U} \prec \mathcal{V}$ we mean that $\mathcal{U}$ refines $\mathcal{V}$.)

To define the generalized oscillation $\omega_{f}$ of $f$, put

$$
\omega_{f}(G)=\inf \left\{\frac{1}{n}: n \in \omega, \exists V \in \mathcal{G}_{n} \quad \text { with } \quad f(G) \subset V\right\}
$$

for a subset $G$ of $X$.

The generalized oscillation $\omega_{f}$ of $f$ is defined as follows:

$$
\omega_{f}(x)=\inf \left\{\omega_{f}(O): O \in \mathcal{B}(x)\right\},
$$

where $\mathcal{B}(x)$ stands for a base of open neighbourhoods of $x$.

Remark. We should notice that the generalized oscillation for a metrizable range space does not reduce to the oscillation. However, our generalized oscillation satisfies many good properties which the oscillation has.

\section{Generalized oscillation and continuity points of functions}

Theorem 3.1. Let $X$ be a topological space and $Y$ be a weakly developable space. Let $f: X \rightarrow Y$ be a function. Then $f$ is continuous at $x \in X$ if and only if $\omega_{f}(x)=0$.

Pro of. Suppose $f: X \rightarrow Y$ is continuous at $x \in X$. Let $\epsilon>0$. There is $n \in \omega$ with $1 / n<\epsilon$. Let $G \in \mathcal{G}_{n}$ be such that $f(x) \in G$. The continuity of $f$ at $x$ implies that there is $O \in \mathcal{B}(x)$ with $f(O) \subset G$. Thus $\omega_{f}(O) \leq 1 / n<\epsilon$; i.e., $\omega_{f}(x) \leq \omega_{f}(O)<\epsilon$. Since $\epsilon>0$ was arbitrary $\omega_{f}(x)=0$.

Suppose now that $\omega_{f}(x)=0$. For every $n \in \omega$, there is $O_{n} \in \mathcal{B}(x)$ and $G_{n} \in \mathcal{G}_{n}$ with $f\left(O_{n}\right) \subset G_{n}$.

Let $n \in \omega$. Since $\omega_{f}(x)<1 / n$, there must exist

$$
O_{n} \in \mathcal{B}(x) \quad \text { with } \quad \omega_{f}\left(O_{n}\right)<\frac{1}{n} .
$$

By the definition of $\omega_{f}\left(O_{n}\right)$, there is $k \in \omega$ such that $f\left(O_{n}\right) \subset V$ for some $V \in \mathcal{G}_{k}$ and $\omega_{f}\left(O_{n}\right) \leq 1 / k<1 / n$; i.e., $k>n$. Thus $V \in \mathcal{G}_{n}$ since $\mathcal{G}_{k} \prec \mathcal{G}_{n}$ for $k>n$. 


\section{LUBICA HOLÁ - ZBIGNIEW PIOTROWSKI}

To prove that $f$ is continuous at $x$, let $H$ be an open set in $Y$ with $f(x) \in H$. By the above, $f(x) \in G_{n} \in \mathcal{G}_{n}$ for every $n \in \omega$. By the assumption on $Y$, there is $m \in \omega$ with $\cap\left\{G_{j}: 1 \leq j \leq m\right\} \subset H$. Thus

$$
f\left(\cap\left\{O_{j}: 1 \leq j \leq m\right\}\right) \subset H .
$$

Proposition 3.2. Let $X$ be a topological space and $Y$ be a weakly developable space. Let $f: X \rightarrow Y$. The generalized oscillation $\omega_{f}$ is upper semicontinuous.

Proof. Let $x \in X$ and $\epsilon>0$. If $\omega_{f}(x)=1$, then we are done. Suppose that $\omega_{f}(x)<1$. There must exist $O \in \mathcal{B}(x)$ with $\omega_{f}(O)<\omega_{f}(x)+\epsilon$. For every $z \in O$ we have

$$
\omega_{f}(z) \leq \omega_{f}(O)<\omega_{f}(x)+\epsilon .
$$

Thus $\omega_{f}$ is upper semicontinuous at $x$.

TheOrem 3.3 (see GP1 for developable spaces). Let $X$ be a topological space and $Y$ be a weakly developable space. Let $f: X \rightarrow Y$ be a function. Then the set $C(f)$ of the points of continuity of $f$ is a $G_{\delta}$-set.

Proof. For every $n \in \omega$, put

$$
\Omega_{n}=\left\{x \in X: \omega_{f}(x)<\frac{1}{n}\right\} .
$$

Of course, $\Omega_{n}$ is open since by the above proposition $\omega_{f}$ is upper semicontinuous. By Theorem 3.1

so we are done.

$$
C(f)=\cap\left\{\Omega_{n}: n \in \omega\right\},
$$

As we mentioned above every weakly developable space has a $G_{\delta}$-diagonal. The following result shows that to have a $G_{\delta}$-diagonal is a necessary condition on $Y$ in Theorem 3.3.

TheOREm 3.4. Let $Y$ be a topological space with the following property:

$(\triangle)$ Given a topological space $X$ and a function $f: X \rightarrow Y$, then the set $C(f)$ of the points of continuity of $f$ is a $G_{\delta}$-set in $X$.

Then $Y$ has a $G_{\delta}$-diagonal.

To prove the above Theorem, we will use the construction from BLL] and the following simple fact.

Lemma 3.5. Suppose $Y=\prod\{Y(n): n \in \omega\}$, where each $Y(n)$ has property $(\triangle)$. Then so does $Y$. 


\section{CONTINUITY POINTS OF FUNCTIONS}

Proof. For each $n \in \omega$ let $p_{n}: Y \rightarrow Y(n)$ be the projection. Suppose $X$ and $f: X \rightarrow Y$ are given as in $(\triangle)$. It is easy to realize that

$$
C(f)=\cap\left\{C\left(p_{n} \circ f\right): n \in \omega\right\} .
$$

By the assumption, each of the composite mappings $p_{n} \circ f: X \rightarrow Y(n)$ has a $G_{\delta}$-set of the points of continuity $C\left(p_{n} \circ f\right)$. So, we are done.

Pro of of The or em 3.4. We will use the proof of [BLL, Proposition 2.10]. Let $S=\{1 / n: n \geq 1\} \cup\{0\}$ with the usual topology. Let $X=Y^{2} \times S$. Let

$$
H=\left\{\frac{1}{2} n: n \geq 1\right\} \quad \text { and } \quad L=\left\{\frac{1}{(2 n-1)}: n \geq 1\right\} .
$$

We define a function $f: X \rightarrow Y^{2}$ as follows:

$$
\begin{aligned}
& f(x, y, t)=(x, y) \quad \text { if } \quad(x, y, t) \in Y^{2} \times H, \\
& f(x, y, t)=(y, x) \quad \text { if } \quad(x, y, t) \in Y^{2} \times L, \\
& f(x, x, 0)=(x, x), \quad \text { if } \quad x \neq y . \\
& f(x, y, 0)=(x, y) \quad
\end{aligned}
$$

It is easy to verify that $f$ is continuous at every point of $A$, where

$$
A=\left(Y^{2} \times\left\{\frac{1}{n}: n \geq 1\right\}\right) \cup(\triangle \times\{0\}),
$$

and $\triangle$ is a diagonal of $Y^{2}$. By Lemma 3.5, also $Y^{2}$ has the property $(\triangle)$. Thus $A=C(f)$ must be a $G_{\delta}$-set in $X$. Because $\triangle \times\{0\}$ is a $G_{\delta}$-set in $A, \triangle \times\{0\}$ is also a $G_{\delta}$-set in $Y^{2} \times S$ and also in $Y^{2} \times\{0\}$. Thus, $\triangle$ is a $G_{\delta}$-set in $Y^{2}$.

The following example shows that to have a $G_{\delta}$-diagonal, it is not sufficient to guarantee that the set of continuity points of every function is a $G_{\delta}$ set. Thus the condition of a weak developability of $Y$ in Theorem 3.3 is essential.

ExAMPLE 3.6. Let $Y$ be the Michael line (the real line with the isolated irrationals and the rationals having their usual neighbourhoods) and $X=R$ with the usual topology. Let $f: X \rightarrow Y$ be the identity mapping. It is easy to verify that $C(f)=Q$, where $Q$ is the set of rational numbers, i.e., $C(f)$ is not a $G_{\delta}$-set in $X$. The Michael line is a submetrizable non-developable space $([\mathrm{Gr}])$. Thus it is not weakly developable. (The Michael line is a paracompact space and, by [AAC, Proposition 2.6], a paracompact weakly developable space is metrizable.)

An easy modification of the proof of $\mathrm{R}$. B ols t e in [Bo] shows that his result also works if a range space is a non-discrete weakly developable space.

Proposition 3.7. Let $X$ be an almost-resolvable space and $Y$ be a non-discrete weakly developable space. Let $F$ be a $F_{\sigma}$-set in $X$. Then there is a function $f: X \rightarrow Y$ such that $C(f)=X \backslash F$. 


\section{LUBICA HOLÁ - ZBIGNIEW PIOTROWSKI}

We say that a topological space is almost-resolvable [Bo] if it is the countable union of sets with empty interiors. A topological space is resolvable [He, $\mathrm{V}]$ if it is the union of two disjoint dense sets. E. Hewitt $[\mathrm{He}$ showed that first countable spaces without isolated points and locally compact spaces without isolated points are resolvable. N. V. Veli ch k o $[\mathrm{V}]$ proved that even $k$-spaces without isolated points are resolvable.

Clearly, a resolvable space is almost-resolvable, and an almost-resolvable space has no isolated points. Note that if a space $X$ contains a dense set which is a countable union of sets with empty interiors, then $X$ is almost-resolvable. Thus a separable space with no isolated points is almost-resolvable.

Using the same idea as in GP2, we can prove the following lemma.

Lemma 3.8. Let $X$ be a topological space and $Y$ be a weakly developable nondiscrete space. Let $A$ be a dense set in $X$. Then $A$ is a $G_{\delta}$-set in $X$ if and only if there is a function $f: X \rightarrow Y$ with $C(f)=A$.

Proof. As $A$ is a $G_{\delta}$-set in $X$ we may write $A=\cap A_{n}$, where each $A_{n}$ is open, $A_{n+1} \subset A_{n}$ for each $n$ and $A_{1}=X$. Let $y \in Y$ be a non-isolated point in $Y$. The first countability of $Y$ implies that there is a sequence of different points $\left\{y_{n}: n \in \omega\right\}$ convergent to $y$ such that $y_{n} \neq y$ for every $n \in \omega$. Define $f: X \rightarrow Y$ as follows:

$$
\begin{array}{ll}
f(x)=y & \text { if } \quad x \in A, \\
f(x)=y_{n} & \text { if } \quad x \in A_{n} \backslash A_{n+1} .
\end{array}
$$

It is easy to verify that $C(f)=A$.

Say that a topological space $X$ is Volterra [GP1] if $C(f) \cap C(g)$ is dense in $X$ whenever $f, g: X \rightarrow R$ are two functions for which $C(f)$ and $C(g)$ are dense in $X$.

Of course, every Baire space is a Volterra space and there are Volterra spaces which are not of second Baire category GP3, hence not Baire. It was proved in GL that every metrizable Volterra space is Baire.

The following result generalizes [GP2, Theorem 1].

Proposition 3.9. For any topological space $X$, the following are equivalent:

(1) $X$ is Volterra;

(2) for each pair $A, B$ of dense $G_{\delta}$-subsets of $X$, the set $A \cap B$ is dense;

(3) for each pair $Y, Z$ of weakly developable spaces and each pair $f: X \rightarrow Y$ and $g: X \rightarrow Z$ of functions for which $C(f)$ and $C(g)$ are dense in $X$, the set $C(f) \cap C(g)$ is dense;

(4) for each pair $f, g$ of functions from $X$ to $Y$, where $Y$ is a fixed nondiscrete weakly developable space, with $C(f), C(g)$ dense in $X$, the set $C(f) \cap C(g)$ is dense in $X$. 
Proof.

$(1) \Leftrightarrow(2)$ Theorem 1 in GP2.

$(2) \Rightarrow(3)$ By Theorem 3.3, $C(f)$ and $C(g)$ are $G_{\delta}$-sets in $X$.

$(3) \Rightarrow(4)$ Clear.

$(4) \Rightarrow(2)$ Suppose $A, B$ are dense $G_{\delta}$-subsets of $X$. By Lemma 3.8, there are functions $f, g: X \rightarrow Y$ for which $C(f)=A$ and $C(g)=B$.

\section{Continuity points of quasicontinuous functions}

In [P1] the following question was posed:

"Assume $X$ is a Baire space. What are "large" spaces $Y$ such that every quasicontinuous function $f: X \rightarrow Y$ has $C(f) \neq \emptyset$ ?"

We say that a function $f$ from a topological space $X$ to a topological space $Y$ is quasicontinuous at $x$ of $X([\mathrm{~N},[\mathrm{P} 1])$ if for every open neighbourhood $V$ of $f(x)$ and each open neighbourhood $U$ of $x$ there exists a non-empty open set $W \subset U$ such that $f(W) \subset V$. If $f$ is quasicontinuous at every point of $X$, we say that $f$ is quasicontinuous.

Of course, by "large" spaces in the above question we understand neither metrizable nor having a countable base since for such spaces $Y$ every quasicontinuous function $f$ from a Baire space into $Y$ has $C(f)$ a dense $G_{\delta}$-set in $X$.

Theorem 4.1. Let $X$ be a Baire space and $Y$ be a weakly developable space. Let $f: X \rightarrow Y$ be a quasicontinuous function. Then the set $C(f)$ of the points of continuity of $f$ is a dense $G_{\delta}$-set.

Proof. For every $n \in \omega$, put $\Omega_{n}=\left\{x \in X: \omega_{f}(x)<1 / n\right\}$. The upper semicontinuity of $\omega_{f}$ implies that every $\Omega_{n}$ is open. Now, we prove that $\Omega_{n}$ is a dense set for every $n \in \omega$.

Let $V$ be a nonempty open set in $X$. Let $x \in X$. There is $G \in \mathcal{G}_{2 n}$ with $f(x) \in G$. The quasicontinuity of $f$ at $x$ implies that there is a nonempty open set $W$ such that

Thus

$$
W \subset V \text { and } f(W) \subset G \text {. }
$$

$$
\omega_{f}(W) \leq \frac{1}{2 n}<\frac{1}{n} .
$$

For every $z \in W$ we have $\omega_{f}(z) \leq \omega_{f}(W)<1 / n$, i.e., $\emptyset \neq W \subset V \cap \Omega_{n}$.

Baireness of $X$ implies that $\cap\left\{\Omega_{n}: n \in \omega\right\}$ is a dense set. Since

$$
C(f)=\cap\left\{\Omega_{n}: n \in \omega\right\},
$$

we are done. 
In $[\mathrm{KKM}]$ we can find a better solution of the above question. However, KKM does not guarantee $G_{\delta}$-set of points of continuity of quasicontinuous functions.

In the last part of this section, we will mention the result concerning continuity points of separately continuous functions which generalizes [P1, Theorem 3]. The proof of our result uses an idea of the generalized oscillation.

Given topological spaces $X, Y$ and $Z$, a function $f: X \times Y \rightarrow Z$ is said to be quasicontinuous with respect to the variable $x$ at $(p, q) \in X \times Y[\mathrm{P} 1]$ if for every neighbourhood $W$ of $f(p, q)$ and for every neighbourhood $U \times V$ of $(p, q)$, there exists a neighbourhood of $p, U^{\prime} \subset U$ and a nonempty open set $V^{\prime} \subset V$ such that for all $(x, y) \in U^{\prime} \times V^{\prime}$, we have $f(x, y) \in W$.

Theorem 4.2 ([P1] for $Z$ Moore). Let $X$ be a first countable space, $Y$ be a Baire space and $Z$ be a regular weakly developable space. If $f: X \times Y \rightarrow Z$ has

a) all sections $f_{x}$ quasicontinuous and

b) all sections $f_{y}$ continuous, then $C(f) \cap(\{x\} \times Y)$ is a dense $G_{\delta}$ subset of $\{x\} \times Y$ for every $x \in X$.

Proof. Let $x \in X$. We prove that $\left\{y \in Y: \omega_{f}(x, y)=0\right\}$ is a dense $G_{\delta^{-}}$set in $\{x\} \times Y$, then by our Theorem 3.1, we are done.

Let $\left\{\mathcal{G}_{n}: n \in \omega\right\}$ be a weak development on $Z$.

Let $n \in \omega$. Put

$$
H_{n}=\left\{y \in Y: \omega_{f}(x, y)<\frac{1}{n}\right\} .
$$

We show that $H_{n}$ is an open dense set in $Y$. Let $G$ be a nonempty open set in $Y$. Let $y \in G$. Let $V$ be such an element from $\mathcal{G}_{n+1}$ that $f(x, y) \in V$. By the result in [P2], $f$ is quasicontinuous with respect to the variable $x$. Thus there is an open neighbourhood $U$ of $x$ and a nonempty open set $H \subset G$ such that $f(u, v) \in V$ for every $(u, v) \in U \times H$, so $\omega_{f}(U \times H)<1 / n$. Thus also, $\omega_{f}(u, v)<1 / n$ for every $(u, v) \in U \times H$; i.e., $H \subset H_{n}$. Thus, $G \cap H_{n} \neq \emptyset$, i.e., $H_{n}$ is dense in $Y$.

To prove that $H_{n}$ is open, let $y \in H_{n}$. The set $W=\left\{(u, v): \omega_{f}(u, v)<1 / n\right\}$ is open in $X \times Y$ since $\omega_{f}$ is upper semicontinuous. Thus, there are open neighbourhoods $U_{x}, U_{y}$ of $x$ and $y$, respectively, with $U_{x} \times U_{y} \subset W$; i.e., $U_{y} \subset H_{n}$. Thus $H_{n}$ is open.

\section{Continuity points of functions with closed graphs}

In this part we study continuity points of functions with closed graphs. Results in this direction can be found also in [D, PS]. The following theorems show that to guarantee $G_{\delta}$-set of continuity points for functions with closed graphs, we do not need the assumption of $G_{\delta}$-diagonal for a range space. 
TheOrem 5.1. Let $X$ be a topological space and $Y$ be a p-space. Let $f: X \rightarrow Y$ be a function with a closed graph. Then the set $C(f)$ is a $G_{\delta^{-}}$set in $X$.

P r o of. Let $\left\{\mathcal{U}_{n}: n \in \omega\right\}$ be a sequence of families of open subsets of $\beta Y$ such that

(i) each $\mathcal{U}_{n}$ covers $Y$;

(ii) for each $y \in Y, \cap\left\{s t\left(y, \mathcal{U}_{n}\right): n \in \omega\right\} \subset Y$.

Further, let $\left\{\mathcal{H}_{n} n \in \omega\right\}$ be a sequence of families of open subsets of $\beta Y$ such that $\left\{\bar{G}^{\beta Y}: G \in \mathcal{H}_{n}\right\}$ refines $\mathcal{U}_{n}$ for every $n \in \omega$. For every $n \in \omega$, put

$$
\Omega_{n}=\left\{x \in X: \exists O \in \mathcal{B}(x), \exists H \in \mathcal{H}_{n} \quad \text { with } \quad f(O) \subset H\right\},
$$

where $\mathcal{B}(x)$ stands for a base of neighbourhoods of $x$.

Of course, $\Omega_{n}$ is open in $X$ for every $n \in \omega$. We claim that

$$
C(f)=\cap\left\{\Omega_{n}: n \in \omega\right\} .
$$

The inclusion $C(f) \subset \cap\left\{\Omega_{n}: n \in \omega\right\}$ is trivial. Now, we prove the opposite one. Let $x \in \cap\left\{\Omega_{n}: n \in \omega\right\}$ and suppose that $f$ is not continuous at $x$. There must exist an open set $L \subset Y$ such that $f(x) \in L$ and $f(G) \cap(Y \backslash L) \neq \emptyset$ for every $G \in \mathcal{B}(x)$.

For every $n \in \omega$, let $O_{n} \in \mathcal{B}(x)$ and $H_{n} \in \mathcal{H}_{n}$ be such that $f\left(O_{n}\right) \subset \mathcal{H}_{n}$.

The family

$$
\mathcal{L}=\left\{{\overline{f\left(O \cap O_{n}\right) \cap(Y \backslash L)}}^{\beta Y}: O \in \mathcal{B}(x), n \in \omega\right\}
$$

is a centered family of closed sets in $\beta Y$, thus $\cap \mathcal{L} \neq \emptyset$. Let $y \in \cap \mathcal{L}$. Then for every $n \in \omega, y \in \operatorname{st}\left(f(x), \mathcal{U}_{n}\right) .\left(y \in \cap \mathcal{L} \subset{\overline{f\left(O_{n}\right) \cap(Y \backslash L)}}^{\beta Y} \subset{\overline{H_{n}}}^{\beta Y}\right.$ and $\left\{\bar{G}^{\beta Y}: G \in \mathcal{H}_{n}\right\}$ refines $\mathcal{U}_{n}$.)

Thus $y \in Y$ and, of course, $y \in Y \backslash L$. It is easy to verify that $(x, y) \in \overline{G(f)}$, where $G(f)$ is the graph of $f$, a contradiction.

From the proof of Theorem 5.1, we can deduce that every function with a closed graph with values in a locally compact space has an open set of points of its continuity. However, this result is already known.

Corollary $5.2([\mathrm{PS}])$. Let $X$ be a topological space and $Y$ be locally compact. If $f: X \rightarrow Y$ has a closed graph, then the set $C(f)$ is an open subset of $X$.

A topological space $Y$ is a $w \Delta$-space if there is a sequence $\left\{\mathcal{G}_{n}: n \in \omega\right\}$ of open covers of $Y$ such that for each $y \in Y$ if $y_{n} \in \operatorname{st}\left(y, \mathcal{G}_{n}\right)$ for each $n \in \omega$, then the set $\left\{y_{n}: n \in \omega\right\}$ has a cluster point in $Y$.

The notions $p$-space and a $w \Delta$-space are independent in general. However, in the class of submetacompact spaces these two notions coincide $([\mathrm{Gr}])$. 


\section{LUBICA HOLÁ - ZBIGNIEW PIOTROWSKI}

Of course, every countably compact space is a $w \Delta$-space. Every countably compact space which is not a $k$-space is an example of a $w \Delta$-space which is not a $p$-space. Gruenhage's space [Gr, Example 2.17] is an example of a $p$-space which is not a $w \Delta$-space (see $\mathrm{Gr}$ ).

Theorem 5.3. Let $X$ be a first countable topological space and $Y$ be a $w \triangle$ -space. Let $f: X \rightarrow Y$ be a function with a closed graph. Then the set $C(f)$ of continuity points of $f$ is a $G_{\delta}$-set in $X$.

Proof. Let $\left\{\mathcal{G}_{n}: n \in \omega\right\}$ be a sequence of open covers of $Y$ such that if $y_{n} \in \operatorname{st}\left(y, \mathcal{G}_{n}\right)$ for each $n \in \omega$, then the set $\left\{y_{n}: n \in \omega\right\}$ has a cluster point in $Y$. For every $n \in \omega$, put

$$
\Omega_{n}=\left\{x \in X: \exists O \in \mathcal{B}(x), \exists V \in \mathcal{G}_{n} \quad \text { with } \quad f(O) \subset V\right\}
$$

as above.

We claim that $C(f)=\cap\left\{\Omega_{n}: n \in \omega\right\}$. Of course, the inclusion

$$
C(f) \subset \cap\left\{\Omega_{n}: n \in \omega\right\}
$$

is clear. Now, we prove the opposite one. Let $x \in \cap\left\{\Omega_{n}: n \in \omega\right\}$ and suppose that $f$ is not continuous at $x$. There must exist an open set $L$ in $Y$ such that $f(x) \in L$ and $f(G) \cap(Y \backslash L) \neq \emptyset$ for every $G \in \mathcal{B}(x)$. Let $\left\{G_{n}: n \in \omega\right\}$ be a decreasing countable base of neighbourhoods of $x$. For every $n \in \omega$ let $O_{n} \in \mathcal{B}(x)$ and $V_{n} \in \mathcal{G}_{n}$ be such that $f\left(O_{n}\right) \subset V_{n}$. Let $n \in \omega$. Thus also $f\left(O_{n} \cap G_{n}\right) \subset V_{n}$ and by the assumption there is $y_{n} \in f\left(O_{n} \cap G_{n}\right) \backslash L$. For every $n \in \omega, f(x) \in V_{n}$ and also $y_{n} \in V_{n}$, i.e., $y_{n} \in \operatorname{st}\left(f(x), \mathcal{G}_{n}\right)$. Since $Y$ is a $w \triangle$-space, the set $\left\{y_{n}: n \in \omega\right\}$ has a cluster point $y \in Y$. Of course, $y \in Y \backslash L$. It is easy to verify that $(x, y) \in \overline{G(f)}=G(f)$ and $y \neq f(x)$, a contradiction.

The following example shows that the conditions of a $p$-space as well as of a $w \Delta$-space in Theorems 5.1 and 5.3 are essential.

ExAmple 5.4. Let $X, Y, f$ be the same as in Example 3.6. Then, of course, $X$ is first countable, $f$ is a function with a closed graph, and $Y$ is neither $p$-space nor $w \Delta$ space (see $[\mathrm{Gr}$, Corollary 3.4]). As we mentioned in Example 3.6, the set $C(f)$ is not a $G_{\delta^{-}}$set.

Now, we use our Theorem 5.1 to generalize Raja's result and to offer a simple proof of his result.

A topological space is a Baire space provided countable collections of open dense subsets have a dense intersection (equivalently nonempty open subsets are of the 2nd Baire category). A topological space is a hereditarily Baire space provided every nonempty closed subset is a Baire space. 
Theorem 5.5. Let $X$ be a hereditarily Baire $p$-space and $f: X \rightarrow Y$ be a continuous bijective map. If $f^{-1}$ has a dense set of points of continuity, then $Y$ contains a dense Baire subspace. In particular, $Y$ is a Baire space.

Proof. The function $f^{-1}: Y \rightarrow X$ has a closed graph since $f: X \rightarrow Y$ is continuous and $Y$ is a Hausdorff space. So, the graph $G(f)$ of $f$ is closed in $X \times Y$, i.e., also $G\left(f^{-1}\right)$ is closed in $Y \times X$. By Theorem 5.1, the set $C\left(f^{-1}\right)$ of the points of continuity of $f^{-1}$ is a $G_{\delta^{-}}$set in $Y$ since $X$ is a $p$-space. Put

$$
H=C\left(f^{-1}\right) \text {. }
$$

Let $\left\{G_{n}: n \in \omega\right\}$ be a sequence of open sets in $Y$ such that

$$
H=\cap\left\{G_{n}: n \in \omega\right\} .
$$

Then $g=f^{-1} \uparrow H$ is a continuous function from $H$ to $X$.

$$
g(H)=f^{-1}(H)=f^{-1}\left(\cap\left\{G_{n}: n \in \omega\right\}\right)=\cap\left\{f^{-1}\left(G_{n}\right): n \in \omega\right\}=L .
$$

Thus, $L$ is a $G_{\delta^{-}}$set in $X$. The set $\bar{L}$ is a Baire space by the assumption and $L$ is a dense $G_{\delta}$-set in $\bar{L}$, thus $L$ is also a Baire space. $f \uparrow L$ is a homeomorphism between $L$ and $H$. Thus also $H$ is a Baire space.

Remark. We can see from the above proof that if $X$ is a Cech-complete space, then we obtain Raja's result which claims that $Y$ contains a dense Cech-complete subspace.

\section{REFERENCES}

[AAC] ALLECHE, B.-ARHANGELSKII, A. V.-CALBRIX, J.: Weak developments and metrization, Topology Appl. 100 (2000), 23-38.

[Bo] BOLSTEIN, R.: Set of points of discontinuity, Proc. Amer. Math. Soc. 38 (1973), 193-197.

[BLL] BURKE, V.-LUTZER, D.-LEVI, S.: Functional characterizations of certain p-spaces, Topology Appl. 20 (1985), 161-165.

[CA] CALBRIX, J.-ALLECHE, B.: Multifunctions and Čech-complete spaces, in: Proc. of the 8th Prague Topological Symposium, Prague, Czech Republic, August 18-24, 1996 (P. Simon, ed.), Topology Atlas, Prague, 1997, pp. 30-36.

[D] DOBOŠ, J.: On the set of points of discontinuity for functions with closed graphs, Čas. pěst. mat. 110 (1985), 60-68.

[E] ENGELKING, R.: General Topology. PWN, Warsaw, 1977.

[Gr] GRUENHAGE, G.: Generalized metric spaces, in: Handbook of Set-Theoretic Topology (K. Kunen, J. Vaughan, eds.), North Holland, Amsterdam, 1984, pp. 423-501.

[GL] GRUEnhaGE, G.-LUTZER, D.: Baire and Volterra spaces, Proc. Amer. Math. Soc. 128 (2000), 3115-3124.

[GP1] GAULD, D. B.-PIOTROWSKI, Z.: On Volterra spaces, Far East J. Math. Sci. 1 (1993), 209-214.

[GP2] GAULD, D. B.-GREENWOOD, S.-PIOTROWSKI, Z.: On Volterra spaces II, Annals New York Academy of Sciences 806 (1996), 169-173. 


\section{LUBICA HOLÁ - ZBIGNIEW PIOTROWSKI}

[GP3] GAULD, D. B.-GREENWOOD, S.-PIOTROWSKI, Z.: On Volterra spaces III, Topology Proc. 23 (1998), 167-182.

[He] HEWITT, E.: A problem of set-theoretic topology, Duke Math. J. 10 (1943), 309-333.

[Ke] KELLEY, J.: General Topology. New York, 1955.

[KKM] KENDEROV, P. S.-KORTEZOV, I. S.-MOORS, W. B.: Continuity points of quasicontinuous mappings, Topology Appl. 109 (2001), 321-346.

[N] NEUBRUNN, T.: Quasi-continuity, Real Anal. Exchange 14 (1988), 259-306.

[Ra] RAJA: Absolute first Borel class topological spaces (preprint).

[P1] PIOTROWSKI, Z.: Separate and joint continuity in Baire groups, Tatra Mt. Math. Publ. 14 (1998), 109-116.

[P2] PIOTROWSKI, Z.: Quasi-continuity and product spaces, in: Proc. Intern. Conf. Geom. Topology, Warsaw, 1978, PWN, Warsaw, 1980, pp. 349-352.

[PS] PIOTROWSKI, Z.-SZYMANSKI, A.: Closed graph theorem: Topological approach, Rend. Circ. Mat. Palermo (2) 37 (1988), 88-99.

[V] VELICHKO, N. V.: Theory of resolvable spaces, Mat. Zametki 19 (1976), 109-114.

Received December 12, 2007

\author{
L'ubica Holá \\ Mathematical Institute \\ Slovak Academy of Sciences \\ Štefánikova 49 \\ SK-814-73 Bratislava \\ SLOVAKIA \\ E-mail: hola@mat.savba.sk \\ Zbigniew Piotrowski \\ Department of Mathematics \\ Youngstown State University \\ Youngstown, Ohio 44555-0001 \\ U.S.A. \\ E-mail: zpiotr@math.ysu.edu
}

